\title{
Do Training Programs Change the Attitude of Health Care Providers toward Venous Thromboembolism Prophylaxis?
}

\author{
Ebtisam Bakhsh ${ }^{1}$, Anas H Barakat ${ }^{2}$ and Emad R Issak ${ }^{3 *}$ \\ ${ }^{1}$ College of Medicine, Department of Clinical Sciences, Princess Nourah Bint \\ Abdulrahman University, Riyadh, Saudi Arabia \\ ${ }^{2}$ Consultant of Internal Medicine, Director of Clinical Audit and General Director of \\ Health Affairs of Riyadh, Riyadh, Saudi Arabia \\ ${ }^{3}$ Specialist of Internal Medicine and Research Director, Nova Clinics, Egypt \\ *Corresponding Author: Emad R Issak, Specialist of Internal Medicine and \\ Research Director, Nova Clinics, Zahraa Al-Maadi, Cairo, Egypt. \\ E-mail: dr.emad.r.h.issak@gmail.com
}

\begin{abstract}
Background: Venous thromboembolism (VTE) is a universal health problem` which causes mortality. Prophylaxis of venous thromboembolism is useful in decreasing mortality and morbidity rates. There is a worldwide deficiency in awareness about venous thromboembolism.

Aims: To investigate the knowledge, attitude, and practice of venous thromboembolism prophylaxis between medical staff in Saudi medical community before and after a training workshop.

Materials and Methods: This study includes three medical professions from 20 rural hospitals in Saudi Arabia. A survey was used to record their answers. Data analyzed using suitable statistical methods.

Results: Training has a significant $(\mathrm{p}<0.001)$ positive impact on the awareness of the availability of VTE guidelines $(39.9 \%$ and $90.9 \%$ ) before and after training, respectively. Also, the availability of risk assessment form has been increased ( $p<0.001)$ after training (37.5\% and 89.1\%) before and after training, respectively. Participants' prescription of VTE prophylaxis for patients upon admission was significantly ( $\mathrm{p}$-value $<0.001$ ) increased $(23.2 \%$ and $67.6 \%$ ) before and after training, respectively. Before training, most of the hospitals had no mechanical prophylaxis devices (84.3\%). However, after training this percent decreased (38.9\%) significantly $(\mathrm{p}<0.001)$. The most prevalent barrier to implement thromboprophylaxis guidelines fully was that each physician had his own approach to provide thromboprophylaxis $(50.2 \%)$. That was decreased significantly ( $p<0.001)$ after training $(35.6 \%)$.

Conclusion: Training and education are very important to help increase the awareness and to change attitudes of health care providers toward VTE prophylaxis. Addressing the barriers toward VTE prophylaxis implementation is necessary. Health care providers are willing to help in planning and implementation and this is an excellent strategy for implementation of VTE prophylaxis.
\end{abstract}

Keywords: VTE; VTE Prophylaxis; VTE Guidelines; Training; Attitude

\section{Abbreviations}

CDC: Centre for Disease Control and Prevention; GCS: Graduated Compression Stockings; IPC: Intermittent Pneumatic Compression; LMWH: Low Molecular Weight Heparin; VTE: Venous Thromboembolism

\section{Introduction}

Worldwide, venous thromboembolism (VTE) is a major public health challenge responsible for morbidity, disability, mortality as well as a diminished health-related quality of life [1-3]. It is a pre-

Citation: Emad R Issak, et al. "Do Training Programs Change the Attitude of Health Care Providers toward Venous Thromboembolism Prophylaxis?". Acta Scientific Medical Sciences 4.9 (2020): 137-144. 
ventable cause of morbidities and mortalities in hospitalized patients $[4,5]$.

A systematic review of the literature about VTE in high-income, middle-income and low-income countries reported an annual incidence rates ranging from 0.75 to 2.69 per 1000 individuals in the population. This incidence is higher in elderly people ( $\geq 70$ years) as 2 - 7 per $1000[5,6]$.

Hospital-acquired VTE accounts for $75 \%$ of all VTE-related deaths, whereas half of the cases occur after medical illness or hospitalization for surgery [4]. Centre for Disease Control and Prevention (CDC) estimated mortality resulting from VTE in the United States (U.S.) to be almost 60,000 - 100,000 annually [8,9]. In Saudi Arabia, it was found that VTE represented $1.9 \%$ of hospital mortality in 1 year [10].

Preventive strategies was proven cost-effective and could decrease adverse outcomes of VTE including morbidities and mortalities $[5,11,12]$. In the hospital settings, it was shown that effective thromboprophylaxis practices could prevent hospital-acquired VTE $[5,12,13]$. Thromboprophylaxis encompasses the use of pharmacological agents including LMWH, warfarin and unfractionated heparin; and mechanical devices including venous foot pump, graduated compression stockings (GCS) and intermittent pneumatic compression (IPC). Both strategies are available for the surgical and medical patient $[14,15]$.

The American College of Chest Physicians (ACCP) VTE Prophylaxis guideline, which is adopted in Saudi hospitals, described the stratification of the risk of VTE in general and abdominal-pelvic surgical patients. They recommended just early ambulation for a very low $(<0.5 \%)$ risk for VTE. For patients at low risk for VTE $(\sim 1.5 \%)$, they suggested intermittent pneumatic compression (IPC). For patients at moderate risk for VTE $(\sim 3 \%)$, they suggested low-molecular-weight heparin (LMWH), low-dose unfractionated heparin, or IPC. For patients at high risk for VTE ( 6\%), they recommended LMWH or low-dose unfractionated heparin. In these patients, they suggested adding mechanical prophylaxis with elastic stockings or IPC to pharmacologic prophylaxis. For patients at high risk for VTE undergoing abdominal or pelvic surgery for cancer, they recommend extended-duration, postoperative, pharmacologic prophylaxis with LMWH over limited-duration prophylaxis [16].
However, VTE prophylaxis for high-risk hospitalized patients is underutilized, and guidelines implementations are sparse in most hospitals globally [17-20]. As it was reported that $71.8 \%$ of physicians do not perform VTE risk assessment of their patients before admission and a higher percent of $81.2 \%$ did not follow any clinical guidelines on thromboprophylaxis use [14].

The gap between guideline and practice has been reported in the ENDORSE study which found that more than $50 \%$ of patients should have received VTE thromboprophylaxis, but in fact only $50 \%$ of them actually received it [19]. Also, the IMPROVE registry has reported that only $60 \%$ of at risk patients were receiving any prophylaxis [2].

A study in Saudi Arabia revealed that there is improper implementation of the ACCP VTE Prophylaxis guideline, which is prominent in medical patients. This lack in practice was attributed to underestimation of risk and absence of a formal policy for VTE prophylaxis. Authors also suggested that health care providers should be aware of VTE prophylaxis [10].

Another study stated that although $60.5 \%$ of cases received some form of VTE prophylaxis, only $38.4 \%$ of them received ACCP-recommended VTE prophylaxis. They concluded that the VTE prophylaxis guideline was not properly implemented and was underutilized [21].

Hence, attitude and knowledge of VTE prophylaxis are very important to improve mortality and morbidity related to VTE [22]. Thus, in this study, we aimed to assess knowledge and attitude of health care providers toward VTE prophylaxis before and after training workshops about it.

\section{Materials and Methods}

This cohort-selection cross-sectional study was conducted in two phases. The first one was just before workshops about VTE prophylaxis in January 2017. The second phase was conducted in May 2017. The participants of this study were of three different professions; physicians either consultants, specialists or residents, nurses, and pharmacists. The participants were from the same hospitals. The study included 594 health care providers in the first phase and 506 in the second phase. Only 427 participant responded for both surveys, 167 responded only for before-training survey and 49 responded only to after-training survey, table 1. 
Do Training Programs Change the Attitude of Health Care Providers toward Venous Thromboembolism Prophylaxis?

\begin{tabular}{|l|c|c|c|}
\hline & $\begin{array}{c}\text { Responded for } \\
\text { both surveys }\end{array}$ & $\begin{array}{c}\text { Only before- } \\
\text { training } \\
\text { survey }\end{array}$ & $\begin{array}{c}\text { Only after- } \\
\text { training } \\
\text { survey }\end{array}$ \\
\hline Nurses & 141 & 89 & \\
\hline Specialists & 169 & & 6 \\
\hline Residents & 96 & 57 & \\
\hline Pharmacists & 10 & 21 & \\
\hline Consultants & 11 & & 43 \\
\hline Total & 427 & 167 & 49 \\
\hline
\end{tabular}

\begin{tabular}{|l|c|c|c|c|}
\hline \multirow{2}{*}{ Barriers } & \multicolumn{2}{|c|}{ Before training } & \multicolumn{2}{c|}{ After training } \\
\cline { 2 - 5 } & Number & $\mathbf{\%}$ & Number & $\%$ \\
\hline Nurses & 230 & $38.7 \%$ & 141 & $27.9 \%$ \\
\hline Specialists & 169 & $28.5 \%$ & 175 & $34.6 \%$ \\
\hline Residents & 153 & $25.8 \%$ & 96 & $19 \%$ \\
\hline Pharmacists & 31 & $5.2 \%$ & 10 & $2 \%$ \\
\hline Consultants & 11 & $1.9 \%$ & 54 & $16.6 \%$ \\
\hline
\end{tabular}

Table 2: Participants professions.

Table 1: Participants professions responded for each survey.

The participants involved in this study were from 20 rural hospitals in Riyadh, KSA. A semi-structured questionnaire of 10 questions was used to investigate demographics, knowledge, and attitude of the health care participants. We investigated the barriers to implement thromboprophylaxis guidelines in the hospitals where participants work, many answers options were included and the participant was able to choose more than one answer. In addition, we asked about the help that participants could provide to optimize thromboprophylaxis in their hospitals. They were able to choose more than one choice.

The workshops' program was titled with (how to prevent venous thromboembolism and deep blood clots) and were conducted in General Directorate of Health Affairs in Riyadh, king Salman bin Abdul-Aziz Hospital and Obstetrics and Gynecology Hospital - King Saud Medical City in Riyadh city. The workshops included lectures and case scenarios.

Data were statistically described in terms of frequencies and percentages. Comparison between before and after workshop surveys was done using Chi-square test. The exact test was used instead when the expected frequency is less than five. A p-value less than 0.05 is considered statistically significant. All statistical calculations were done using computer program IBM SPSS (Statistical Package for the Social Science; IBM Corp, Armonk, NY, USA) Release 22 for Microsoft Windows.

\section{Results and Discussion}

Before training, the more dominant participants were nurses with a percent of $38.7 \%$ (230/594); followed by specialists who represented $28.5 \%(169 / 594)$. Other professions are shown in table 2 .
The awareness of the availability of VTE prophylaxis and management guidelines in the hospitals was significantly ( $\mathrm{p}$-value < 0.001) increased after the workshops. Before the training, only 237 (39.9\%) were aware; however, after the training workshops 460 (90.9\%) were aware (Figure 1).

The availability of VTE risk assessment form in hospitals the participants work in was significantly, (p-value $<0.001)$ increased after the workshops. Before the training, only 223 (37.5\%) demonstrated that the risk assessment form was available; however, this percentage was increased after the training workshops to be 451 (89.1\%) (Figure 1).

Participants' prescription of VTE prophylaxis for patients upon admission was significantly (p-value $<0.001$ ) increased after the workshops; mentioned only by 138 (23.2\%) before the training, however, after the training workshops it was prescribed by 342 $(67.6 \%)$ of respondents (Figure 1).

The presence of mechanical VTE prophylaxis devices in hospitals was significantly ( $\mathrm{p}$-value $<0.001$ ) increased after the workshops; mentioned only by $93(15.7 \%)$ of respondents before the training; however, after the training workshops this figure increased significantly to be 309 (61.1\%) of respondents (Figure 1).

\section{Barriers to thromboprophylaxis guidelines implementation}

Before training, the most frequently mentioned barrier was that each physician had his own approach to provide thromboprophylaxis and prescribed thromboprophylaxis on an individual basis, this barrier mentioned by $50.2 \%$ of participants. After training, this barrier was significantly ( $\mathrm{p}$-values $<0.001$ ) decreased, see table 3 for details about all barriers.

It worthwhile mentioning that there is a new barrier increased after training, which is the resistance in some areas or by some physicians. It was mentioned by only $7.1 \%$ before training and by $22.1 \%$ after training, ( $\mathrm{p}$-value $<0.001)$. 


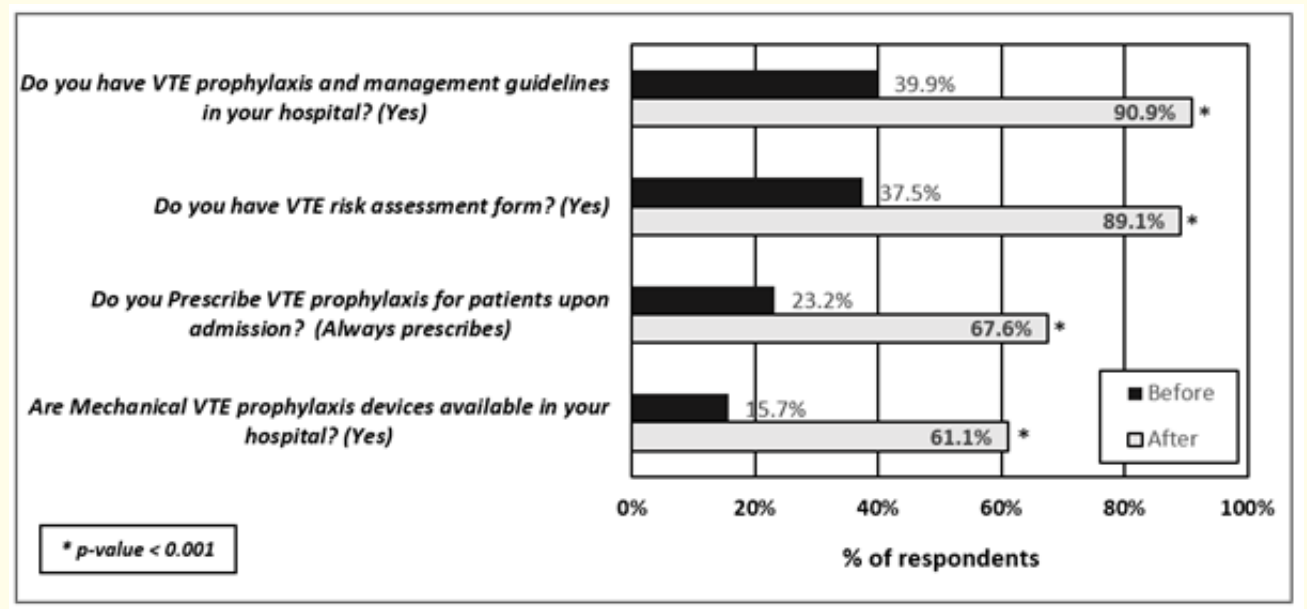

Figure 1: Availability of VTE prophylaxis guidelines and tools.

\begin{tabular}{|c|c|c|c|c|c|}
\hline Barriers & \multicolumn{2}{|c|}{ Before training } & \multicolumn{2}{|c|}{ After training } & P-value \\
\hline 1. Hospital started to make guideline policy and procedure & 2 & $0.3 \%$ & 3 & $0.6 \%$ & 0.666 \\
\hline $\begin{array}{l}\text { 3. Each physician has their own approach to providing thromboprophylaxis } \\
\text { and prescribes thromboprophylaxis on an individual basis }\end{array}$ & 298 & $50.2 \%$ & 180 & $35.6 \%$ & $<0.001$ \\
\hline 5. Physician cannot agree on a uniform policy & 22 & $3.7 \%$ & 34 & $6.7 \%$ & 0.033 \\
\hline $\begin{array}{l}\text { 6. Hospital leader/or physician are not aware of the importance of VTE preven- } \\
\text { tion }\end{array}$ & 30 & $5.1 \%$ & 35 & $6.9 \%$ & 0.238 \\
\hline 7. We don't have hospital support/resources for that & 108 & $18.2 \%$ & 73 & $14.4 \%$ & 0.111 \\
\hline
\end{tabular}

Table 3: Barriers to VTE prophylaxis before and after training.

Before training, most of them (49.2\%) were ready to provide templates of thromboprophylaxis guidelines; however, this percent reduced significantly ( $\mathrm{p}$-value $<0.001)$ after training $(35.2 \%)$. In addition, there were $48 \%$ chose that they could provide literature summarizing the evidence for thromboprophylaxis or for successful implementation strategies; however, this percent reduced significantly ( $\mathrm{p}$-value $<0.001$ ) after training (35.2\%).
There were $45.1 \%$ of participants had the ability to provide education and $41.9 \%$ were able to provide examples of tools to help strengthen the message. These two offers did not change significantly ( $\mathrm{p}$-values $>0.05$ ). Whereas $41.6 \%$ mentioned that, they could share methods and tools to conduct local audits of prophylaxis use. That was reduced significantly ( $p$-value $<0.001$ ) to $26.5 \%$ after training (See table 4 ). 
Do Training Programs Change the Attitude of Health Care Providers toward Venous Thromboembolism Prophylaxis?

\begin{tabular}{|c|c|c|c|c|c|}
\hline \multirow{2}{*}{ Helps offered } & \multicolumn{2}{|c|}{ Before training } & \multicolumn{2}{|c|}{ After training } & \multirow{3}{*}{$\begin{array}{c}\text { P-value } \\
0.212\end{array}$} \\
\hline & Number & $\%$ & Number & $\%$ & \\
\hline $\begin{array}{l}\text { 1. Provide examples of tools to help reinforce the message (i.e. pocket cards, } \\
\text { algorithms) }\end{array}$ & 249 & $41.9 \%$ & 232 & $45.8 \%$ & \\
\hline 2. Provide templates of thromboprophylaxis/guidelines. & 292 & $49.2 \%$ & 178 & $35.2 \%$ & $<0.001$ \\
\hline 3. Share methods and tools to conduct local audits of prophylaxis use & 247 & $41.6 \%$ & 238 & $47.0 \%$ & 0.079 \\
\hline 5. Share examples of successful initiatives at other hospitals & 246 & $41.4 \%$ & 134 & $26.5 \%$ & $<0.001$ \\
\hline 6. Provide education in person & 268 & $45.1 \%$ & 204 & $40.3 \%$ & 0.123 \\
\hline
\end{tabular}

Table 4: Willingness to help to optimize VTE prophylaxis.

\section{Discussion}

The current study showed that training has a significant positive impact on the awareness of the availability of VTE guidelines and on the availability of risk assessment forms. Also, the prescription of VTE prophylaxis for patients upon admission was significantly increased after the training. In addition, the availability of mechanical prophylaxis devices was increased significantly after the training. The most prevalent barrier to implement thromboprophylaxis guidelines fully was that each physician had his own approach to provide thromboprophylaxis; however, that was decreased significantly by training.

The VTE prophylaxis is still suboptimal, due to poor compliance, this return to the lack of knowledge and lack of hospital guidelines $[13,23]$. Even if thromboprophylaxis is applied, the guidelines are not followed $[19,20]$. Therefore, we aimed to investigate the knowledge and attitude of health care personnel before and after training workshops so, we can improve its practice and find gaps that we should fill in order to make improvements.

It was reported that thromboprophylaxis practice in hospitalized patients was suboptimal worldwide $[19,20]$. In the present study, most of the health care providers before training workshop (76.8\%) occasionally or did not prescribe VTE prophylaxis for patients, while those who always prescribe the prophylaxis were $23.2 \%$ only. This reflects the weak practice of VTE prophylaxis, it was found that thromboprophylaxis practice was $83.5 \%$ in one study [13], this is high percent compared to ours. However, in the current study, the effectiveness of training workshop is shown as the percent of participants who always prescribe VTE prophylaxis for patients has been significantly increased (67.6\%).
In the present study, the presence of mechanical VTE prophylaxis devices in hospitals was significantly increased after the workshops; this reflects that the lack of knowledge and practice of VTE prophylaxis can affect the issue. The commonly used devices that were reported by the health care staff were Graduate compression stockings and pneumatic compression device. In a study established in China, it was found that mechanical prophylaxis was effective according to the medical staff participants of the study; also, they reported that the most effective one was Graduate compression stockings [24]. Although the effectiveness of using mechanical prophylaxis was found, most of our participants before training confirmed lacking these devices in their hospitals, this can be attributed to a lack of awareness of strong evidence of the devices' efficiency, and hence limited mechanical devices are of less use and availability in the hospitals of the participants.

In a study by Ekwere., et al. [13], it was found that the majority of physicians did not carry out VTE risk assessment. In our study, before training, $37.5 \%$ of participants said that there was no VTE risk assessment form, compared to $89.1 \%$ after training workshops which reflect the effectiveness of a training in changing the attitudes.

Following thromboprophylaxis clinical guidelines are necessary for good thromboprophylaxis practice; it was found that the percent of participants who did not follow any guidelines in is ranged from $40 \%$ to $81.2 \%[13,25]$. In addition, the lack of awareness of thromboprophylaxis clinical guidelines is mentioned in some studies and ranged from $27.5 \%$ to $60 \%[13,20,26]$. The results of the current study showed that less than the half of participants have thromboprophylaxis clinical guidelines before the training; however, this percent increased significantly to $90.9 \%$ after the training. 
The most prevalent barriers for implementing thromboprophylaxis guidelines in our study were; each physician has his own approach to providing thromboprophylaxis and prescribes thromboprophylaxis on an individual basis, there was no approached VTE prevention systemically in their organization, implementation of prophylaxis plan was slow and there were no hospital support or resources for that. Having personal approaches for thromboprophylaxis and lack of standardized ones seems to be a big problem not only in Saudi Arabia but also in other countries, in a China study, it was reported that more than of medical staff chose nonstandard approaches for VTE prophylaxis in ICU this could be attributed to the deficiency in knowledge of guidelines [13]. Off course, there are differences in barriers between studies in different areas of the World $[13,27,28]$ and these differences may be attributed to the difference in culture, priorities, and availability to practice VTE prophylaxis.

Scaglione., et al. found that identifying barriers for changes in the practice of prophylaxis was one of many reasons that led to increasing in the use of VTE prophylaxis in surgical patients [29]. In our study, after training, these previously mentioned barriers have been reduced significantly which led to change in attitude and awareness toward the use of VTE prophylaxis in hospitals.

However, one of the barriers has been increased after training which is "There is a resistance in some areas or by some physicians". We think that this increase is due to the variation in the sample structure before and after the training. Nurses' number decreased and consultants' number increased. In addition, the need for a customized guideline (Saudi one) may be behind that.

Our study revealed the high attitude of health care providers toward thromboprophylaxis and they were ready to assist their hospitals to optimize VTE prophylaxis. The highest percent of participants desired to provide templates of thromboprophylaxis guidelines; this is a very good step to practice thromboprophylaxis as thromboprophylaxis guidelines is critical for good thromboprophylaxis practice. Scaglione., et al. reported that pocket guidelines were one of the several strategies that increased the use of VTE prophylaxis in surgical patients from $64 \%$ to $97 \%$ [29].

Education is very important as it was reported that the first strategy to enhance VTE compliance rates was increasing awareness of health care providers about VTE prophylaxis through periodic educational sessions [30].
Also, the results of our study showed that high percent expressed their ability to provide literature summarizing the evidence for thromboprophylaxis for successful implementation strategies. However, the percent who could provide literature-summarizing evidence of VTE prophylaxis decreased over the time. That was because a good proportion already provided literature-summarizing evidence of VTE prophylaxis after the first training.

A good percent of health care providers had the ability to provide education. These results reflect the high attitude of the participants toward VTE prophylaxis practice and improvements in their facilities.

The majority of the participants in one study suggested that the use of locally designed clinical guidelines on VTE prophylaxis and frequent educational programs would increase awareness on VTE [13]. Also, Cohn., et al. reported that implementation of a multifaceted VTE prophylaxis improvement program that included education and dissemination of a decision support tool resulted in increasing in VTE prophylaxis by $25 \%$ after 12 months [31]. In a systemic review, it was found that application of many strategies to improve VTE prophylaxis is more effective than the application of only one strategy [30].

One of the advantage of this current study is being comparative over time, conducted in the same hospitals with enough sample size. However, one limitation of the study was that not all the participants of the first survey were included in the second one, thus the structure of the sample according to professions was changed.

The results of that current study make us believe that it can be generalizable as it showed that the training is an effective tool in changing attitudes toward VTE prophylaxis that is reflected in the attitude toward using VTE prophylaxis and the availability of VTE prophylaxis tools. Moreover, the results of this study make us learn that involving the health-care professionals themselves is an important step in the success of implementation of any guideline.

We believe that VTE prophylaxis utilization can be improved by continuous education and follow-up. The availability of summarized guideline schematics at hand is an important step for the success of its implementation.

\section{Conclusion}

Training and education are very important to help increase the awareness and to change attitudes of health care providers toward 
VTE prophylaxis. Addressing the barriers toward VTE prophylaxis implementation is necessary. Health care providers are willing to help in planning and implementation and this is an excellent strategy for implementation of VTE prophylaxis.

\section{Conflict of Interest}

Authors declared they have no conflicts of interest.

\section{Bibliography}

1. DHHS. "The Surgeon General's Call to Action to Prevent Deep Vein Thrombosis and Pulmonary Embolism". Office of the Surgeon General. U.S. Department of Health and Human Services. Washington, DC (2011).

2. Tapson VF., et al. "Venous thromboembolism prophylaxis in acutely ill hospitalized medical patients: findings from the International Medical Prevention Registry on Venous Thromboembolism". Chest 132.3 (2007): 936-945.

3. Ruppert A., et al. "Economic burden of venous thromboembolism: a systematic review". Journal of Medical Economics 14.1 (2011): 65-74.

4. Cohen AT., et al. "Venous thromboembolism (VTE) in Europe. The number of VTE events and associated morbidity and mortality". Thrombosis and Haemostasis 98 (2007): 756-764.

5. ISTH Steering Committee for World Thrombosis Day. Thrombosis: "a major contributor to the global disease burden". Journal of Thrombosis and Haemostasis 12.10 (2014): 1580-1590.

6. Heit JA. "Epidemiology of venous thromboembolism". Nature Reviews Cardiology 12.8 (2015): 464-474.

7. Saxena A., et al. "Safety and efficacy of low-molecular-weight heparins in prophylaxis of deep vein thrombosis in postoperative/ICU patients: A comparative study". Journal of Natural Science, Biology, and Medicine 4.1 (2013): 197-200.

8. CDC. "Data and Statistics on Venous Thromboembolism". Centers for Disease Control and Prevention (2015).

9. Beckman MG., et al. "Venous thromboembolism: a public health concern". American Journal of Preventive Medicine 38.4 (2010): S495-S501.

10. Essam AE., et al. "Venous thromboembolism-related mortality and morbidity in King Fahd General Hospital, Jeddah, Kingdom of Saudi Arabia". Annals of Thoracic Medicine 6.4 (2011): 193-198.
11. Linda MD., et al. "Economic Evaluation of Enoxaparin as Postdischarge Prophylaxis for Deep Vein Thrombosis (DVT) in Elective Hip Surgery". Value in Health 3 (2008): 398-406.

12. Catterick D., et al. "Impact of the national venous thromboembolism risk assessment tool in secondary care in England: retrospective population-based database study". Blood Coagulation and Fibrinolysis 25 (2014): 571-576.

13. Ekwere TA., et al. "Venous thromboembolism: awareness and practice of thromboprophylaxis among physicians in a tertiary-care hospital". International Journal of Medicine and Biomedical Research 4.1 (2015): 14-20.

14. Cohen AT., et al. "ARTEMIS Investigators. Efficacy and safety of fondaparinux for the prevention of venous thromboembolism in older acute medical patients: randomised placebo controlled trial”. British Medical Journal 332 (2006): 325-329.

15. Cayley jr WE. "Preventing deep venous thrombosis in hospital in-patients". British Medical Journal 335 (2007): 147-151.

16. Gould MK., et al. "Prevention of venous thromboembolism: the Seventh ACCP Conference on Antithrombotic and Thrombolytic Therapy". Chest 141 (2012): e227S-e277S.

17. Al-Hameed FM. "Venous thromboembolism prophylaxis: Solutions are in our hands". Annals of Thoracic Medicine 6.3 (2011): 105-106.

18. Serigne AB., et al. "A cross sectional evaluation of venous thromboembolism risk and use of venous thromboembolism prophylaxis in hospitalized patients in Senegal". Archives of Cardiovascular Diseases 104 (2011): 493-501.

19. Cohen AT., et al. "ENDORSE Investigators. Venous thromboembolism, risk and prophylaxis in the acute hospital care setting (ENDORSE study): A multinational cross-sectional study". Lancet 371 (2008):387-394.

20. Kahn SR., et al. "CURVE study investigators. Multicenter evaluation of the use of venous thromboembolism prophylaxis in acutely ill medical patients in Canada". Thrombosis Research 119 (2007): 145-155.

21. Kharaba A., et al. "Venous thromboembolism risks and prophylaxis in King Fahad Hospital, Madinah, Saudi Arabia”. Journal of Epidemiology and Global Health 7.4 (2017): 295-298.

22. Zobeiri M., et al. "Prophylaxis for deep venous thrombosis: Knowledge and practice of surgeons". Pakistan Journal of Medical Sciences 27.1 (2011): 16-20. 
23. Bhatti AM., et al. "Knowledge, Attitude and Practices of Healthcare Providers towards Deep Vein Thrombosis Prophylaxis in Five Teaching Hospitals of Rawalpindi". Journal of Ayub Medical College Abbottabad 24.2 (2012).

24. Tang X., et al. "A Survey of the Knowledge of Venous Thromboembolism Prophylaxis among the Medical Staff of Intensive Care Units in North China". PLOS ONE (2015).

25. Schaden E., et al. "Coagulation Day 2010: an Austrian survey on the routine of thromboprophylaxis in intensive care". Intensive Care Medicine 38.6 (2012): 984-990.

26. Ebrahimpur M., et al. "Knowledge, Attitudes and Practices of Internal Medicine Residents Towards Deep Vein Thrombosis Prophylaxis in a University Hospital in Tehran". Shiraz E Medical Journal 17.12 (2016): e39038.

27. Cook D., et al. "Barriers and facilitators of thromboprophylaxis for medical and surgical Intensive care unit patients: a multicenter survey". The Journal of Critical Care 29 (2014): 471-479.

28. Cabana MD., et al. "Why don't physicians follow Clinical practice guidelines? A framework for improvement". The Journal of the American Medical Association 282 (1999): 1458-1456.

29. Scaglione L., et al. "Implementing guidelines for venous thromboembolism prophylaxis in a large Italian teaching hospital: lights and shadows". Haematologica 90.5 (2005): 678-684.

30. Tooher R., et al. "A systematic review of strategies to improve prophylaxis for venous thromboembolism in hospitals". Annals of Surgery 241 (2005): 397-415.

31. Cohn SL., et al. "Improved use of thromboprophylaxis for deep vein thrombosis following an educational intervention". Journal of Hospital Medicine 1.6 (2006): 331-338.

\section{Assets from publication with us}

- Prompt Acknowledgement after receiving the article

- Thorough Double blinded peer review

- Rapid Publication

- Issue of Publication Certificate

- High visibility of your Published work

Website: www.actascientific.com/

Submit Article: www.actascientific.com/submission.php

Email us: editor@actascientific.com

Contact us: +919182824667 